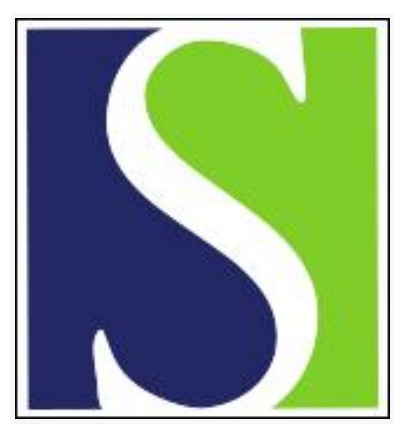

Scand J Work Environ Health 1982;8(3):185-190

https://doi.org/10.5271/sjweh.2481

Issue date: Sep 1982

Ophthamologic effects of man-made mineral fibers.

by Stokholm J, Norn M, Schneider T

This article in PubMed: www.ncbi.nlm.nih.gov/pubmed/6984224

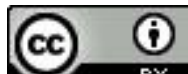




\title{
Ophthamologic effects of man-made mineral fibers
}

\author{
by Jens Stokholm, MD, ${ }^{1}$ Mogens Norn, MD, ${ }^{2}$ Thomas Schneider, $\mathrm{MSc}^{1}$
}

\begin{abstract}
STOKHOLM J, NORN M, SCHNEIDER T. Ophthamologic effects of man-made mineral fibers. Scand $j$ work environ health 8 (1982) 185-190. The effect of man-made mineral fibers on the human eye was investigated in a cross-sectional study of 15 workers exposed to Rockwool $^{\circledast}$ and a matched reference group of 15 people. Eye symptoms, changes in the cellular and mucous content of the conjunctival fluid, break-up time of the precorneal film, the number of microepithelial defects, and the number of dead and degenerated cells on the cornea and bulbar conjunctiva were used as measures of effect. The number of fibers accumulated in the eye and conventional dust sampling methods were used as measures of dose. A significantly higher frequency of eye symptoms related to work conditions $(p<0.001)$ was found among exposed workers. Similarly, the number of microepithelial defects on the medial bulbar conjunctiva increased significantly $(p=0.009)$ after $4 d$ of exposure. Six exposed workers had a pathological increase in the neutrophil count of the conjunctival fluid after $4 \mathrm{~d}$ of exposure, and an increase was seen in only one worker after a weekend free from exposure. Significant correlation was found between microepithelial defects on the medial bulbar conjunctiva and measures of dose $(p<0.01)$. The symptoms and cellular changes can be explained by the assumption that man-made mineral fibers have the same mechanical and reversible effect on the eye as on the skin. The described dose-effect relationship suggests that a much lower hygienic standard is needed for man-made mineral fibers than what has been recommended by the American Conference of Governmental Industrial Hygienists.
\end{abstract}

Key terms: conjunctival cellular changes, conjunctival fluid cytology, dose-effect relationship, epidemiologic study, eye symptoms.

Man-made mineral fibers are regarded as relatively harmless products (5) with an increasing field of application. The effect of these fibers on the skin $(1,4,5,11)$ is well known and is believed to be a mechanical piercing of the epidermis with a secondary release of histamine (11). Irritation of the upper respiratory tract after exposure to man-made mineral fibers has also been reported $(1,9,14)$, whereas epidemiologic investigations have not revealed any harmful effect on the lower respiratory tract so far $(5,15,17)$.

Whenever airborne man-made mineral

1 National Institute of Occupational Health, Hellerup, Denmark.

2 Department of Ophthalmology, Hvidovre Hospital, Copenhagen, Denmark.

Reprint requests to: Dr J Stokholm, National Institute of Occupational Health, Baunegaardsvej 73, DK-2900 Hellerup, Denmark. fibers occur in the environment, the eye will be exposed to them. Since there is a significant correlation between the dose (number of fibers accumulated in the eye during $8 \mathrm{~h}$ ) and the concentration of airborne fibers (13), it is only natural that these fibers should irritate the eye. Eye symptoms caused by such fibers have been reported repeatedly $(1,2,7,12)$, and one case of keratoconjunctivitis with man-made mineral fibers as the possible causative agent has been reported (6). Otherwise, few comments on eye irritation after exposure to man-made mineral fibers can be found in textbooks on toxicology of the eye $(3,8)$.

Against this background, we decided to investigate the effect of man-made mineral fibers on the external eye in connection with a study of the accumulation of fibers in the eyes of workers handling such fibers $\left(\right.$ Rockwool $\left.^{\circledR}\right)$ (13). 


\section{Material and methods}

The investigation was carried out as a cross-sectional study of an exposed group with a matched nonexposed reference group. Matching criteria were age, sex, and smoking habits.

The exposed group included 15 workers (13 men and 2 women) who had been occupied with the production (control of production line), cutting, and packing of Rockwool for at least six months. Users of contact lenses were excluded. One woman in the exposed group was sick and absent from work on the second day of the investigation. The mean age of each group was 38 a (range 19-63 a), and 14 were smokers. The average tobacco consumption was $16 \mathrm{~g} / \mathrm{d}$.

The reference group was selected from the clerical staff of the plant and from employees of the National Institute of Occupational Health. No member of the reference group had been exposed to dust, fibers, or solvents at work or to any large amount of fibers or dust in their spare time during the week before the investigation started.

The investigation program comprised an eye history, based upon a questionnaire and an interview, sampling of conjunctival fluid for quantitative cytology, a general medical examination of the external eye, slit lamp examination, measurement of ruptures in the precorneal film, break-up time, and vital staining of cornea and conjunctiva to reveal any microepithelial defects and dead or degenerated cells. The exposed group went through this program on a Monday, just before resuming work after the weekend, and again four days later, this time at the end of the workday. All the examinations, including the slit lamp examinations, were carried out in a room adjacent to the work area. Those in the reference group went through the same program once, except for the sampling of conjunctival fluid and removal of the mucous thread.

On the basis of information obtained from the questionnaire and in the subsequent interview, we recorded the previous and present use of eye glasses, contact lenses, and protective glasses at work. Similarly, we recorded any previous or present cases of eye infections or minor eye traumas which had been treated by a doctor, the present use of eye medication and smoking habits, as well as any infection in the upper respiratory tract occurring within three weeks prior to the investigation. Finally, the following questions concerning work-related eye symptoms were asked:

1. Have you been troubled by smarting or itching eyes at work?

2. Are your eyes red and irritated at work?

3. Do your eyes water at work?

4. Have you any other eye symptoms related to your work?

If the answer to one of the questions was in the affirmative, the frequency of the symptom - daily, once a week, once a month, or seldom - was registered.

The examination of the external eye was carried out in accordance with ophthalmologic practice (10).

For the counting and classifying of cells in the conjunctival fluid, a quantitative method was used. Conjunctival fluid was aspirated from $\pi \mathrm{mm}^{2}$ conjunctival surface of the left inferior lateral fornix, fixated, and transferred to a slide. After the staining, one of us (MN) searched for, counted, and classified all the cells. Similarly, the amount of mucus was estimated by a semiquantitative method. These procedures have been described in detail earlier $(10)$.

In the exposed group, the coherent mucus in the inferior conjunctival fornix, the mucous thread, was removed from the right eye on the second examination after staining with $0.25 \%$ alcian blue. (Microme no 24, Edward Gurr). The mucous thread was then examined for accumulated manmade mineral fibers. This procedure has also been described in detail earlier (13).

Break-up time was measured twice with a stop-watch after a 10- $\mu$ l drop of $0.125 \%$ fluorescein (resorcinol phthalein) in isotonic sodium chloride had been instilled in the left eye. This procedure was followed by vital staining of the left eye with a $10-\mu 1$ drop of a fluorescein-rose bengal mixture. Approximately $1 \mathrm{~min}$ after the instillation, all the fluoresceinstained microepithelial defects were counted in each of the following areas: lateral bulbar conjunctiva, cornea, medial bulbar conjunctiva, and inferior bulbar 
conjunctiva. Finally, rose bengal-stained degenerated or dead cells were also counted. In each area, the number of defects was subdivided into four groups: $0,1-30,31-100,101-1,000$ and $>1,000$. The values $0,15,65,550$ and 2,000 were used as the average for each group.

A Fisher exact probability test (twosided) was used to test the differences between and within the groups, and the Spearman rank-order correlation test was used to determine any correlation between the groups.

\section{Results}

\section{Questionnaire}

Of those in the exposed group, five reported previous eye infections diagnosed by a doctor in comparison with two in the reference group, and four exposed workers reported eye damage needed to be seen by a doctor in comparison with two in the reference group. Whereas none of the reference group used eye medication at the time of the investigation, three people in the exposed group did. Two of these three people used eye medication almost constantly. The third had visited a firstaid station because of eye irritation, just before the investigation was conducted. The subject reported that the irritation began shortly after "some dust had blown into my eyes." No foreign body had been found. The frequency of eye symptoms related to work conditions is summarized in fig 1.

From the medical interview it appeared that the itching sensation was first and foremost located in the medial bulbar part of the eye. Symptoms were reported to disappear after work, and had usually disappeared by the next morning.

\section{Slit lamp examination}

There was no significant difference between break-up times in the two groups, and the mean values were comparable to other findings (11). Nor was there any significant change in break-up time in the exposed group from Monday morning (mean $37.5 \mathrm{~s}$ ) to Thursday at the end of the workday (mean $40.0 \mathrm{~s}$ ).

The number of epithelial defects, depicted by the fluorescein staining, in- creased significantly $(p=0.009)$ for the medial bulbar conjunctiva after $4 \mathrm{~d}$ of exposure in comparison with those in the reference group (fig 2). Vital staining did not show any other significant differences between and within the groups. However, a slight increase in the number of rose bengal-stained dead or degenerated cells was observed after $4 \mathrm{~d}$ of work (fig 2).

\section{Conjunctival fluid cytology}

In one conjunctival fluid sample an increased number of neutrophils $(11,300)$ and lymphocytes $(1,100)$ was found before exposure to man-made mineral fibers. Moreover one person had a marginally increased number of neutrophils. The other findings were entirely within the normal range (10).

After exposure, only samples from 12 workers were suitable for further examination. A pathologically increased neutrophil count was found in six cases $(p=0.06)$ (range 116-1,530). One sample was accompanied by a pathological increase in the number of columnar epithelial cells, nucleated squamous cells, and keratinized nucleated cells. The corresponding mucous thread and mucous clump sample (13)

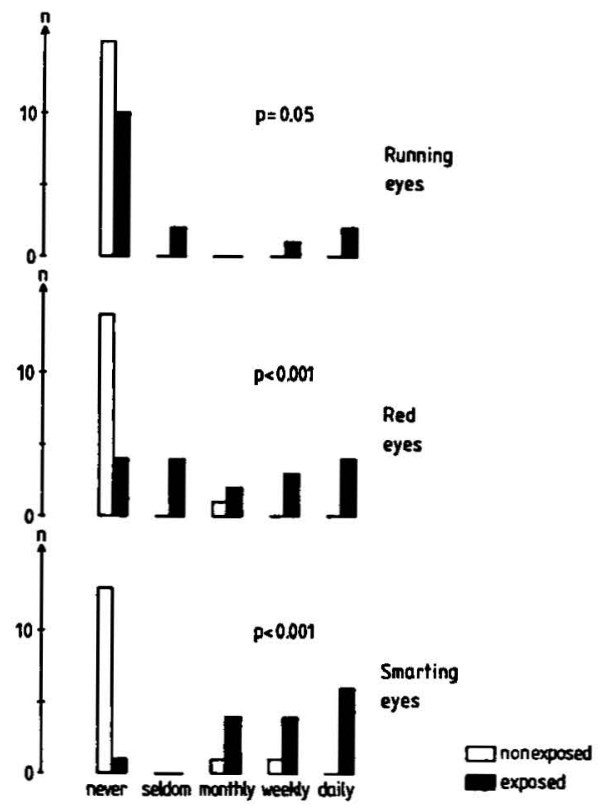

Fig 1. Work-related eye symptoms among 15 subjects exposed to man-made mineral fibers and 15 nonexposed subjects. 
contained the highest number of fibers (250) found after a workday.

In no case did the lymphocyte count increase after exposure, and no esinophilic granulocytes were found.

There was significant correlation between the number of neutrophils and lymphocytes and the number of rose bengal-stained degenerated and dead cells $\left(r_{\mathrm{s}}=0.85\right)$ but not between the number of neutrophils and lymphocytes and the number of fluorescein-stained epithelial defects $\left(r_{\mathrm{s}}=0.12\right)$.

An increased amount of mucus was found in 10 of the 15 conjunctival fluid samples taken on Monday morning and in 8 of the 12 samples taken after exposure. In some of the samples, this increase in mucous content was not accompanied by any subsequent increase in the neutrophilic count.

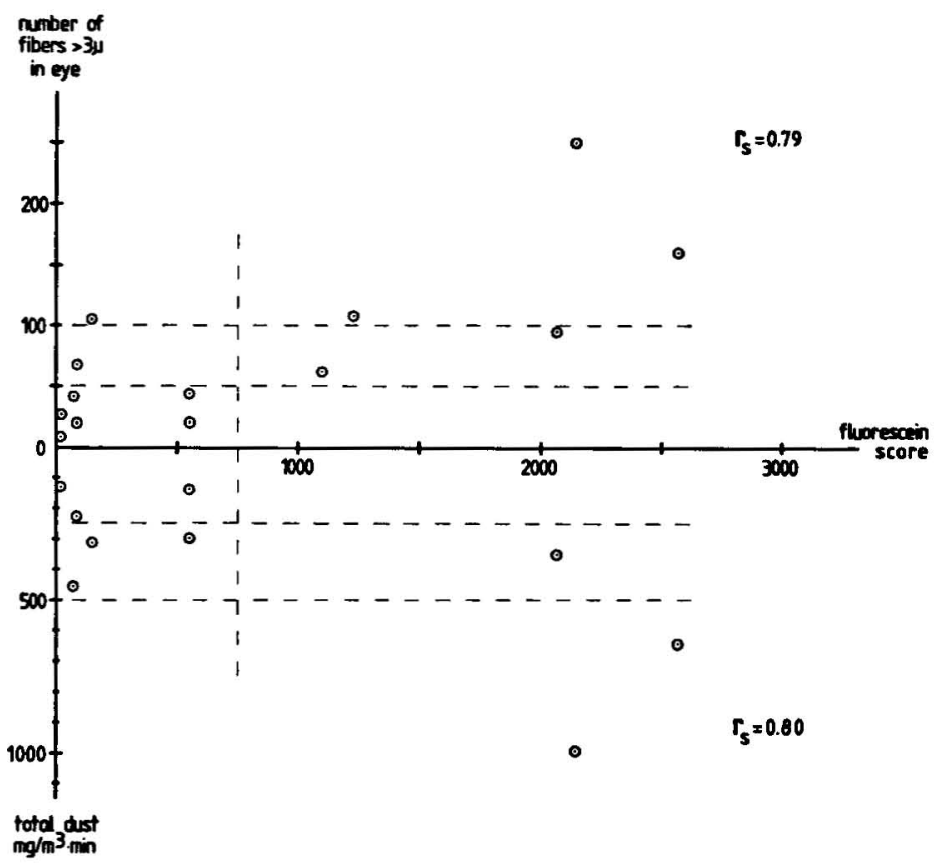

Fig 2. Rose bengal-stained dead cells and fluoresceinstained micropunctated defects of the cornea and the medial bulbar conjunctiva in a group of workers exposed to man-made mineral fibers and in a nonexposed group. Observations of the inferior and lateral bulbar conjunctiva follow a pattern similar to that of the cornea. The exposed group was examined on Monday morning and after the fourth workday. [Abscissa: number of stained minute dots grouped into four categories (see the text) ordinate: number of persons in each group]

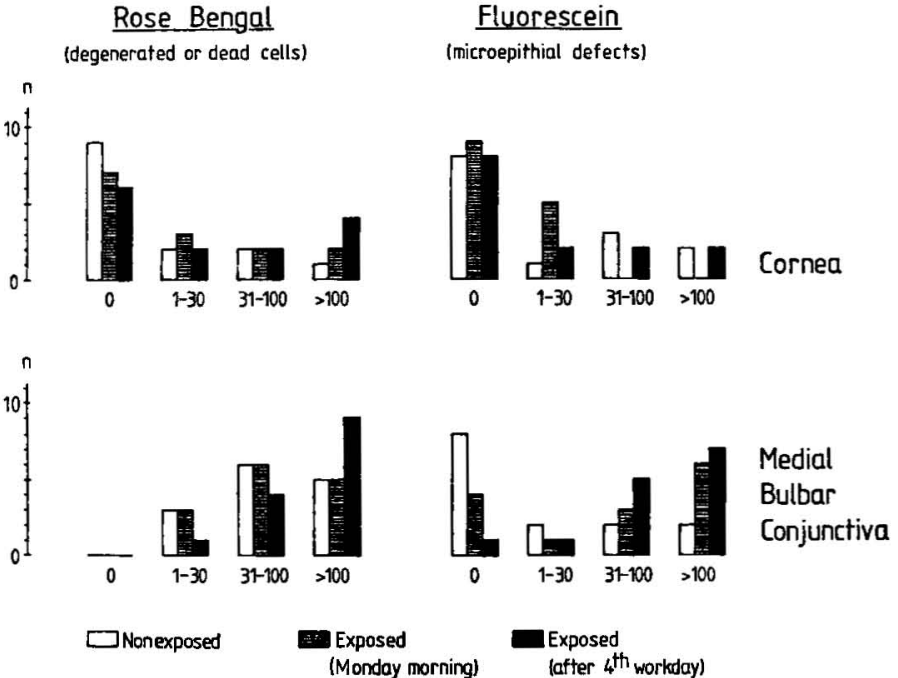

Fig 3. Dose-response relationship. [Abscissa: number of micropunctated fluoresceinstained dots in one eye after the fourth workday; ordinate: number of fibers longer than $3 \mu \mathrm{m}$ that accumulated in sleep and mucous thread and total dust dose sampled on filter $\left(\mathrm{mg} / \mathrm{m}^{3} \times\right.$ exposure time in $\mathrm{min}$ utes) - both during the fourth workday] 


\section{Dose-effect relationship}

We measured the fiber dose by traditional filter methods and by a new method determining the amount of fibers accumulated in the eye (13). Likewise we tested whether a correlation existed between the aforementioned measures of dose and three measures of effect: fluorescein staining, rose bengal staining and conjunctival fluid cytology. There was a significant correlation between the number of fluorescein-stained epithelial defects on the medial bulbar conjunctiva and the number of fibers accumulated in the opposite eye $\left(r_{\mathrm{s}}=0.79, \mathrm{p}<0.01\right)$. There was also a significant correlation between the number of fluorescein-stained epithelial defects and the total airborne dust dose determined by filter sampling $\left(\mathrm{r}_{\mathrm{s}}=0.80, \mathrm{p}<0.01\right)$ (fig 3 ). A nonsignificant correlation was found between the number of rose bengal-stained cells and the number of fibers accumulated in the eye $\left(r_{\mathrm{s}}=0.46, \mathrm{p}=0.07\right)$.

\section{Discussion}

The growing complaints of itching and red eyes, the increase in minor epithelial defects in the conjunctiva, and the increase in the cellular content of conjunctival fluid in a group of workers exposed to man-made mineral fibers can be explained by the assumption that man-made mineral fibers have the same mechanical irritant effect on the eye as on the skin. This assumption is supported by the fact that the conjunctival changes found after exposure were located almost exclusively in the medial bulbar conjunctiva. After deposition, the fibers are arranged parallel to the surface of the bulbus, probably due to the surface tension in the precorneal film (13). The mucus then adheres to the fibers and forms a protective film as the conjunctival fluid is being transported to the medial bulbar area and caruncle. There the mucous thread first curls up and then dries. During this process the free ends of the fibers exceeding $1 \mathrm{~mm}$ in length may point in different directions like a spiked mace and cause mechanical irritation when the eye is blinked or rubbed (13).

The almost identical ophthalmologic findings in the exposed and nonexposed groups of the present study on Monday morning indicate a reversible ophthalmologic effect of man-made mineral fibers in the observed concentrations. However, exposed workers appear to be more at the risk of developing eye infections, and three workers in fact used eye medication at the time of the investigation. Moreover the surprisingly high mucous content in the conjunctival fluid on Monday morning, unaccompanied by other cytological changes, may represent a long-term effect of stimulation of mucous secretion induced by man-made mineral fibers.

The use of eye protection undoubtedly results in fewer symptoms and influences objective findings. Seven people in the exposed group used eye protectors occasionally, and none of them had an increased cellular content in the conjunctival fluid aspirate on Thursday after work, although none of them had used eye protectors on that day.

Other exogenous factors such as age, smoking habits, different types of work, and different fiber materials could also influence symptoms and cellular injuries in the conjunctiva. Age and smoking habits were balanced in the matching of the groups, and the studied group is too small for a detailed analysis of a possible influence of other exogenous factors.

The significant correlation between fibers in the eye and the fluorescein score (fig 3 ) is the first published result which establishes a dose-effect relationship between man-made mineral fibers and an objective measure of acute effect. Different dose levels for predictions of effect can be tested by the application of a validity test (16). For example, if 50 fibers in the eye and $250 \mathrm{mg}$ of fiber dust $/ \mathrm{m}^{3}$ of air $\times$ exposure time in minutes are used as the cut-off levels, the result will be high sensitivity but not very satisfactory specificity $(0.75$ and 0.50 , respectively). If the cut-off levels are increased to $100 \mathrm{fi}$ bers in the eye and $500 \mathrm{mg}$ of fiber $\mathrm{dust} / \mathrm{m}^{3}$ of air $\times$ exposure time in minutes, specificity increases to a satisfactory level ( 0.88 and 1.00 , respectively), but sensitivity becomes unsatisfactorily low $(0.60$ in both cases).

Our observations are based upon a small study population and should therefore be repeated. In any case, the dose-effect relationship suggests a need for a much 
lower hygienic standard for man-made mineral fibers than what has been recommended $(15,17)$ - particularly if work with man-made mineral fibers is carried out without the use of protective eye glasses.

Finally, we would like to emphasize the value of external eye examinations in occupational and environmental health studies. Eye symptoms and various complaints of eye discomfort are often the first signs of new hazards.

\section{Acknowledgment}

The authors gratefully acknowledge the statistical assistance of $\mathrm{Mr} \mathrm{E}$ Holst.

\section{References}

1. Cirla P. Patologia professionale del vetro filato. Med lav 39 (1948) 152.

2. El-Sadik M. Study for eye complaints among workers in glass wool industry. $J$ egypt public health assoc 42 (1967) 53-62.

3. Grant WM. Toxicology of the eye. Charles C Thomas, Springfield, IL 1974. $493 \mathrm{p}$.

4. Heisel EB, Mitchell JH. Cutaneous reaction to fiberglass. Ind med surg 26 (1957) $547-550$.

5. Hill JW. Health aspects of man-made mineral fibres: A review. Ann occup hyg 20 (1977) 161-173.

6. Longley EO, Jones RC. Fiberglass conjunctivitis and keratitis. Arch environ health 13 (1966) 790-793.

7. Lucas JB. The cutaneous and ocular effects resulting from worker exposure to fibrous dust. In: Le Vee WN, ed. Occupational exposure to fibrous glass: Proceedings of a symposium. Washington, DC 1976. (US dept HEW publication no (NIOSH) 76-151).

8. McDonald TO, Shaduck JA. Eye irritation. In: Marzulli FN, Maibach HJ, ed. Advances in modern toxicology, dermatology and pharmacology. Hemisphere Publishing Co, Washington, London 1977, pp $139-191$.

9. Milby TH, Wolf EC. Respiratory tract irritation from fibrous glass inhalation. $J$ occup med 11 (1969) 409-410.

10. Norn MS. External eye: Methods of examination. Scriptor, Copenhagen 1974.

11. Possick PA, Gellen GA, Key MM. Fibrous glass dermatitis. Am ind hyg assoc $\mathrm{j} 31$ (1970) 12-15.

12. Puschkina FK. Data on hygienic assessment of glass wool dust. Gig tr prof 9 (1956) 28-30. (in Russian with an English summary).

13. Schneider T, Stokholm J. Accumulation of fibers in the eyes of workers handling man-made mineral fiber products. Scand $\mathrm{j}$ work environ health 7 (1981) 271-276.

14. Trumper M, Honingsberg A. Localization by fluorescein of fiberglass in throat. $\mathrm{J}$ am med assoc 131 (1946) 1275.

15. US Department of Health, Education and Welfare. Criteria for a recommended standard: Occupational exposure to fibrous glass. Cincinnati, OH 1977. 208 pp. (DHEW (NIOSH) publication no 77-152).

16. Zielhuis RL, Verberk MM. Validity of biological tests in epidemiological toxicology. Int arch occup health 32 (1974) $167-190$.

17. - Man-made mineral fibres: Report of a working party to the advisory committee on toxic substances. Her Majesty's Stationery Office, London 1979. 36 p. (Discussion document).

Received for publication: 22 June 1981 